\title{
Identification of essential genes in cultured mammalian cells using small interfering RNAs
}

\author{
Jens Harborth ${ }^{1, \star}$, Sayda M. Elbashir ${ }^{2, \star}$, Kim Bechert ${ }^{1}$, Thomas Tuschl2,‡ and Klaus Weber ${ }^{1, \neq}$ \\ ${ }^{1}$ Department of Biochemistry and Cell Biology and ${ }^{2}$ Department of Cellular Biochemistry, Max Planck Institute for Biophysical Chemistry, \\ Am Fassberg 11, 37077 Göttingen, Germany \\ *These authors contributed equally to this work \\ ¥Authors for correspondence (e-mail: ttuschl@mpibpc.gwdg.de; office.weber@mpibpc.gwdg.de) \\ Accepted 10 October 2001 \\ Journal of Cell Science 114, 4557-4565 (2001) @ The Company of Biologists Ltd
}

SUMMARY

We report the first RNAi-induced phenotypes in mammalian cultured cells using RNA interference mediated by duplexes of 21-nt RNAs. The 21 gene products studied have different functions and subcellular localizations. Knockdown experiments monitored by immunofluorescence and immunoblotting show that even major cellular proteins such as actin and vimentin can be silenced efficiently. Genes were classified as essential or nonessential depending on impaired cell growth after RNA silencing. Phenotypes also involved altered cell morphology and aberrant mitotic arrest. Among the essential genes identified by RNAi for which such information was previously not available are lamin B1, lamin B2, NUP153, GAS41, ARC21, cytoplasmic dynein, the protein kinase cdk1 and both $\beta$ - and $\gamma$-actin. Newly defined nonessential genes are emerin and zyxin. Several genes previously characterized by other methods such as knockout of murine genes are included as internal controls and gave identical results when RNAi was used. In the case of two nonessential genes (lamin $\mathrm{A} / \mathrm{C}$ and zyxin) RNAi provides a recognizable phenotype.

Our results complete the characterization of the mammalian nuclear lamins. While lamins $\mathrm{A} / \mathrm{C}$ appear as nonessential proteins in the mouse embryo and in RNAi treated cultured cells, the two other lamins, B1 and B2, are now identified as essential proteins. Interestingly the inner nuclear membrane protein emerin, thought to be a ligand of lamin $\mathrm{A} / \mathrm{C}$, is also a nonessential protein in tissue culture cells.

Key words: Functional genomics, Gene silencing, Mammalian cells, Nuclear lamins, RNA interference

\section{INTRODUCTION}

Gene function has been determined traditionally by methods such as deletion of murine genes, the introduction of (mutated and/or dominant negative) transgenes, the molecular characterization of human hereditary diseases, and targeting of genes by antisense techniques (Porter, 1998). Microinjection of an antibody into tissue culture cells (Blangy et al., 1995) and the use of Xenopus oocyte extracts (Desai et al., 1999; Abrieu et al., 2000) can also provide information on protein functions. As the sequencing of the human and murine genomes approaches completion there is an increasing demand for quick, robust and efficient mechanisms to analyse gene function in mammalian cell culture.

RNA interference (RNAi) provides a new approach for elucidation of gene function. RNAi is a sequence-specific, post-transcriptional gene silencing mechanism initiated in animals and plants by the introduction of double stranded RNA (dsRNA) homologous in sequence to the silenced gene (Fire, 1999; Bass, 2000; Cogoni and Macino, 2000; Plasterk and Ketting, 2000; Sijen and Kooter, 2000; Hammond et al., 2001; Matzke et al., 2001; Sharp, 2001; Tuschl, 2001; Waterhouse et al., 2001). RNAi has significantly advanced our understanding of gene function in the nematode $C$. elegans and about one third of the nematode genome has already been subjected to functional analysis by RNAi (Fraser et al., 2000; Gonczy et al.,
2000; Barstead, 2001; Hope, 2001; Kim, 2001; Maeda et al., 2001). Mammalian cells were until recently not amenable to RNAi since use of in vitro transcribed, long dsRNAs (>30 bp) led to activation of a global, sequence unspecific response resulting in blockage of initiation of protein synthesis and mRNA degradation (Bass, 2001). Although RNA interference seems to work in early mouse development (Svoboda et al., 2000; Wianny and Zernicka-Goetz, 2000), it seemed not generally applicable to mammalian cells (Caplen et al., 2000; Ui-Tei et al., 2000).

We recently reported that duplexes of 21-nt RNAs with 2-nt 3' overhang, introduced by transfection into human and other mammalian cultured cells specifically interfered with gene expression and bypassed the sequence independent response of mammalian cells to long dsRNA (Elbashir et al., 2001a). These short RNA duplexes resemble the processing products from long dsRNAs, and are referred to as small interfering RNAs (siRNAs) (Elbashir et al., 2001b). One of the enzymes involved in processing of long dsRNAs has recently been identified and is a member of the RNase III family of nucleases (Bernstein et al., 2001).

We previously documented siRNA-induced silencing of lamin $\mathrm{A} / \mathrm{C}$ in HeLa cells and mentioned that this approach also worked for lamin B1 and the nuclear mitotic apparatus protein NuMA but not for vimentin. We speculated that the negative result with vimentin could be due to the abundance of the 
protein or the particular RNA duplex used (Elbashir et al., 2001a). Here we extend our analysis to a large number of genes in mammalian tissue culture cells. We show that RNAi results in silencing of major cellular proteins such as actin and that vimentin can be effectively silenced by selecting three new RNA duplexes. We report the first phenotypes obtained by RNAi in mammalian cells. Among the essential genes identified are lamin B1, lamin B2, NUP153, GAS41, ARC21, cytoplasmic dynein and the mitotic protein kinase $\operatorname{cdk} 1$, for which such information was not available previously by ablation of murine genes or other approaches.

\section{MATERIALS AND METHODS}

\section{siRNA preparation}

To design target-specific siRNA duplexes, we selected sequences of the type $\mathrm{AA}\left(\mathrm{N}_{19}\right) \mathrm{UU}(\mathrm{N}$, any nucleotide) from the open reading frame of the targeted mRNA, in order to obtain a 21-nt sense and 21-nt antisense strand with symmetric 2-nt $3^{\prime}$ overhangs of identical sequence. We used 2 -deoxythymidines instead of uridine residues in the $3^{\prime}$ overhangs to reduce costs of RNA synthesis and to enhance nuclease resistance. It may also possible to design siRNAs with other sequences in the overhang, or to select siRNAs from the sequences $\mathrm{AA}\left(\mathrm{N}_{21}\right)$ by changing the last two nucleotides of the sense siRNA to TT (the 2-nt overhang of the sense siRNAs is not believed to contribute to target recognition). A selected siRNA sequence was also submitted to a BLAST search against the human genome sequence to ensure that only one gene of the human genome was targeted. 21-nt RNAs were chemically synthesized using Expedite RNA phosphoramidites and thymidine phosphoramidite (Proligo, Hamburg, Germany), deprotected and gel-purified as described (Elbashir et al., 2001a; Elbashir et al., 2001b) or purchased from Dharmacon (Lafayette, $\mathrm{CO}$ ) in deprotected and desalted form.

The accession numbers given below in brackets are from GenBank. The siRNA sequence targeting NuMA (Z11583) was from position 3988-4010 relative to the start codon; GAS41 (NM_006530) 327-349; SV40 T-antigen (62000) 26-48, 639-661; lamin A/C (NM_005572) 608-630; lamin B1 (NM_005573) 672-694; lamin B2 (M94362) 1457-1479 (of the sequence with missing 5'end); LAP2 (NM_003276) 37-59, common region; emerin (NM_000117) 628650; Nup153 (NM_005124) 2593-2615; $\beta$-actin (NM_001101) 972994; $\gamma$-actin (NM_001614) 8-30; ARC21 (AF006086) 181-203; mouse zyxin (X99063) 1357-1379; mouse vinculin (L18880) 29232945; VASP (XM_009141) 1087-1109; vimentin (NM_003380) 346368, 1145-1167, 863-885, 1037-1059; keratin 18 (NM_000224) 11541176; Eg5 (NM_004523) 1547-1569; CENP-E (NM_001813) 944-966; cytoplasmic dynein 1 heavy chain (U53530) 509-531 of partial cDNA; cdk1 (NM_001786) 125-147. As unspecific siRNA control a sequence targeting firefly (Photinus pyralis) luciferase gene (X65324) 153-175 was used. siRNA duplex formation (annealing) was performed as previously described (Elbashir et al., 2001a).

\section{Cell culture and transfection}

Human HeLa SS6 cells and F5 and FR(wt648) rat fibroblast cells (Zerrahn and Deppert, 1993) were grown at $37^{\circ} \mathrm{C}$ in Dulbecco's modified Eagle medium supplemented with $10 \%$ FCS, penicillin and streptomycin. Cells were regularly passaged to maintain exponential growth. The day before transfection, cells were trypsinized, diluted with fresh medium without antibiotics and transferred to 24-well plates $(500 \mu \mathrm{l}$ per well). Transient transfection of siRNAs was carried out using Oligofectamine (Life Technologies). $12 \mu$ I OPTIMEM 1 medium (Life Technologies) and $3 \mu$ l Oligofectamine per well were preincubated for 5-10 minutes at room temperature. During the time for this incubation $50 \mu \mathrm{l}$ OPTIMEM 1 medium were mixed with $3 \mu \mathrm{l}$ siRNA. The two mixtures were combined and incubated for 20 minutes at room temperature for complex formation. After addition of $32 \mu \mathrm{l}$ of OPTIMEM 1 medium to the mixture, the entire mixture was added to the cells in one well resulting in a final concentration of $100 \mathrm{nM}$ for the siRNAs. The addition of $32 \mu$ OPTIMEM 1 medium is optional and was only used to adjust the final culture volume to 600 $\mu 1$. Cells were usually assayed 40-48 hours after transfection, but in some cases also after 70 hours. Specific silencing was confirmed by at least three independent experiments.

\section{Immunofluorescence microscopy, antibodies and immunoblotting}

Immunofluorescence and chromatin staining was performed as described (Elbashir et al., 2001a). Pictures were taken using a Zeiss Axiophot with a F Fluar $40 \times / 1.30$ oil objective and MetaMorph Imaging Software (Universal Imaging Corporation, West Chester, PA) with equal exposure times for the silenced and the control treated cells. For phase-contrast microscopy, cells were mounted in Hepes buffered DMEM medium supplemented with $10 \%$ FCS. Pictures were taken using a Plan-Neofluar $25 \times / 0.8$ objective.

Several antibodies were kindly provided by B. Burke (monoclonal Nup153), W. Deppert (monoclonal SV40 T-antigen 108), M. Osborn (monoclonal lamin A/C clone 636, NuMA, vimentin monoclonal V9, keratin 18 CK2) and J. Wehland (monoclonal ARC21 7H3, VASP 273D4, vinculin, zyxin 164ID4). Commercial antibodies were from Abcam ( $\beta$-actin), Novocastra (lamin B2 clone LN43.2, emerin clone 4G5), Santa Cruz Biotechnology (lamin B1 C-20), Sigma ( $\alpha$-tubulin) and Transduction Laboratories (LAP2 clone 27). The antibody for GAS41 was described previously (Harborth et al., 2000).

For western blotting, transfected cells grown in 24 -well plates, were trypsinized, washed once in ice-cold PBS and harvested. Cells from one well were solubilized in $50 \mu \mathrm{l}$ SDS sample buffer and boiled for 5 minutes. Equal amounts of total protein were separated on 7.5 or $12.5 \%$ polyacrylamide gels and transferred to nitrocellulose. Immunostaining with specific antibodies and peroxidase-conjugated secondary antibodies (Dako, Denmark) diluted 1:20,000 was carried out using the ECL technique (Amersham Pharmacia Biotech). To confirm equal loading, blots were stripped (Re-Blot Western Blot Recycling Kit, Chemicon) and reprobed with the vimentin V9 antibody or in case of vimentin silencing with the $\beta$-actin antibody.

\section{RESULTS}

\section{Selection of target genes}

We examined silencing of 21 genes expressed in cultured mammalian cells by RNA interference with duplexes of 21-nt RNAs (Table 1). These included 18 genes in human HeLa cells, 1 gene in SV40-transformed rat fibroblasts, and 2 genes in mouse $3 \mathrm{~T} 3$ cells. The sequences of the siRNA duplexes were selected from the coding region of the target mRNAs as described in Materials and Methods. In all transfection experiments cultures were transfected in parallel with targetspecific siRNA duplexes and a nonspecific duplex of firefly luciferase from Photinus pyralis (GL2) at the same final concentration as the specific siRNAs tested (Elbashir et al., 2001a). As a further control we used the addition of buffer instead of the volume of siRNA added to the culture. Gene silencing was documented by immunoblotting and immunofluorescence, except in five cases where no suitable antibodies were available. To screen for phenotypes, we looked for impaired cell growth and altered cell morphology monitored by phase microscopy. Aberrant mitotic arrest was visualized using the $\alpha$-tubulin antibody.

The genes selected for this study code for a wide range of 
Table 1. Gene silencing by RNAi: growth arrest and phenotypes in cell culture

\begin{tabular}{|c|c|c|c|c|}
\hline Gene & Silencing* & Essential & Phenotypic change & Gene function by other methods \\
\hline \multicolumn{5}{|l|}{ Nuclear proteins } \\
\hline NuMA & + & + & Apoptotic & $\begin{array}{l}\text { Antibody injection, mutant cDNAs: essential } \\
\text { for mitosis and nuclear formation }\end{array}$ \\
\hline GAS41 & + & + & Arrest & \\
\hline SV40 T-antigen & + & - & & \\
\hline \multicolumn{5}{|c|}{ Nuclear envelope proteins } \\
\hline Lamin A/C & + & - & Emerin displaced & Null mice: nonessential in development; \\
\hline Lamin B1 & + & + & Apoptotic & essential after birth \\
\hline Lamin B2 & + & + & Apoptotic & \\
\hline LAP2 & + & - & & \\
\hline Emerin & + & - & & \\
\hline Nup153 & + & + & & \\
\hline \multicolumn{5}{|c|}{ Cytoskeletal cytoplasmic proteins } \\
\hline$\beta$ Actin & + & + & Blebbing & \\
\hline$\gamma$ Actin & nd & + & Blebbing & \\
\hline ARC21 & + & + & & \\
\hline VASP & + & - & & \\
\hline Vinculin & + & + & & Null mice: embryonic lethal \\
\hline Zyxin & + & - & Loss of stress fibers & \\
\hline Vimentin & + & - & & Null mice: nonessential \\
\hline Keratin 18 & + & - & & Null mice: nonessential \\
\hline \multicolumn{5}{|l|}{ Mitotic proteins } \\
\hline Eg5 & nd & + & Aberrant mitotic, monopolar spindles & $\begin{array}{l}\text { Antibody injection, inhibitor: essential for } \\
\text { centrosome separation }\end{array}$ \\
\hline CENP-E & nd & + & Aberrant mitotic, multipolar spindles & $\begin{array}{l}\text { Antisense and immunodepletion: essential } \\
\text { kinetochore-associated motor }\end{array}$ \\
\hline Cytoplasmic dynein & nd & + & Aberrant mitotic arrest & \\
\hline Cdk1 & nd & + & Premitotic arrest & \\
\hline
\end{tabular}

*Gene silencing was monitored by immunofluorescence and/or immunoblotting; nd, not determined because of lack of a suitable antibody. Silencing was performed in human HeLa cells, except for SV40 T antigen (rat fibroblasts) and vinculin and zyxin (mouse 3T3). A stop in growth was taken to indicate that the protein is essential. For gene function by other methods see text.

Fig. 1. Silencing of SV40 large T antigen in a stably transformed rat fibroblast cell line (F5). Triple fluorescence staining of cells transfected with T-antigen siRNA duplex (A,C,E), and with GL2 luciferase siRNA duplex serving as control (B,D,F). (A,B) Staining with T-antigen specific antibody, (C,D) staining with NuMA specific antibody, (E,F) Hoechst staining of chromatin. The few cells cells in A that are stained for $\mathrm{T}$ antigen were probably nontransfected.

(G) Western blot of cells transfected with $\mathrm{T}$ antigen siRNA (left) or luciferase siRNA (right) probed with $\mathrm{T}$ antigen-specific antibody. The blot was stripped and reprobed with vimentin antibody (bottom). Magnification 480× (A-F).
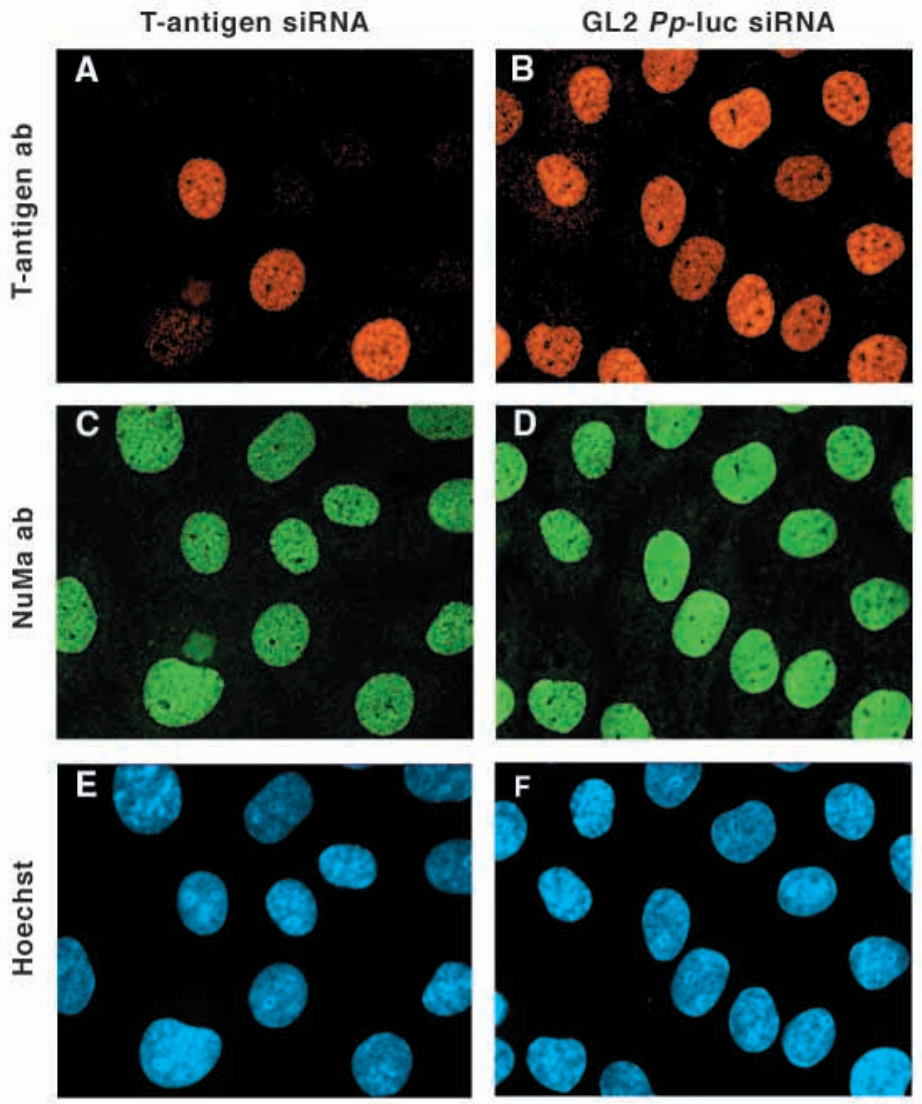
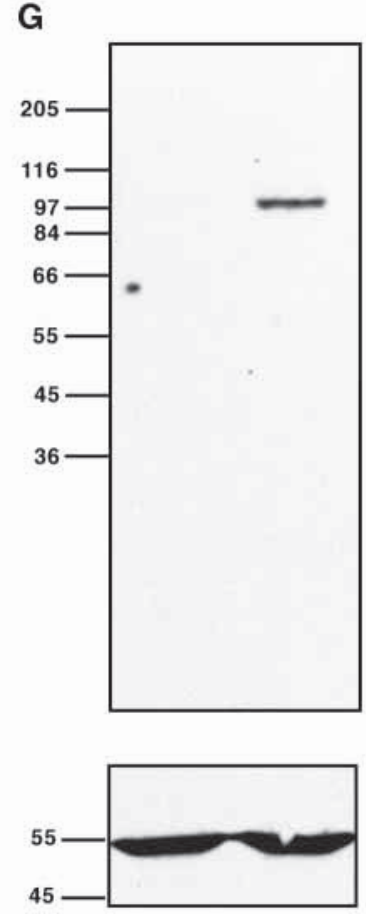
proteins distributed in different cellular compartments. The study includes three nuclear proteins, six proteins of the nuclear envelope, eight cytoplasmic cytoskeletal proteins, three microtubular motor proteins and the protein kinase cdk1. Seven genes, which were already characterized by other methods such as murine knockouts, microinjection of antibodies and transfection by mutant cDNAs served as controls. In these cases, RNAi mediated by siRNAs provided the same result as the earlier studies (Table 1).

\section{Knockdown of nuclear proteins}

Antibody microinjection and transfection with mutant cDNAs showed that the nuclear mitotic apparatus protein NuMA is essential for normal spindles and reformation of nuclei and that NuMA is involved in the early stages of apoptosis (GuethHallonet et al., 1999). Consistent with these results we observed siRNA-induced growth arrest and detected apoptotic cells (Table 1). NuMA was shown to interact with GAS41, a poorly characterized, but highly conserved protein in eukaryotic nuclei (Harborth et al., 2000). Targeting of GAS41 causes growth arrest of HeLa cells, demonstrating for the first time that GAS41 is an essential protein.

As another example of a nuclear protein we tried to target SV40 large $\mathrm{T}$ antigen in transformed rat fibroblasts using two duplexes. Interestingly only one of them resulted in specific silencing (see also vimentin) as demonstrated by immunofluorescence and western blotting (Fig. 1). At least under the conditions used cells continued to grow. It remains to be seen whether this is due to the residual amount of antigen still present or whether $\mathrm{T}$ antigen is indeed nonessential for growth.

\section{Knockdown of proteins of the nuclear envelope}

The nuclear envelope consists of a double membrane, pores, and the lamina, a filamentous meshwork located between the inner nuclear membrane and the peripheral chromatin. We silenced the nuclear lamins $\mathrm{A} / \mathrm{C}, \mathrm{B} 1$ and $\mathrm{B} 2$, the laminaassociated protein LAP2, the inner nuclear membrane protein emerin, and the nuclear pore component Nup153. Knockdown of lamin A/C in HeLa cells did not affect cell growth (Elbashir et al., 2001a), consistent

Fig. 3. Silencing of lamin B1 and lamin B2. HeLa cells were transfected with lamin B1 siRNA $(A, C)$, lamin B2 siRNA (E) or with luciferase siRNA (B,D,F). Cells were either double stained with lamin B1-specific antibody (A,B) and NuMA specific antibody (C,D) or stained for lamin B2 (E,F). (G) Western blot of cells transfected with lamin B1 (left) or luciferase (right) siRNA duplexes using lamin B1 antibody (top). The blot was stripped and re-probed with vimentin antibody to check for equal loading (bottom). Magnification 480× (A-F).
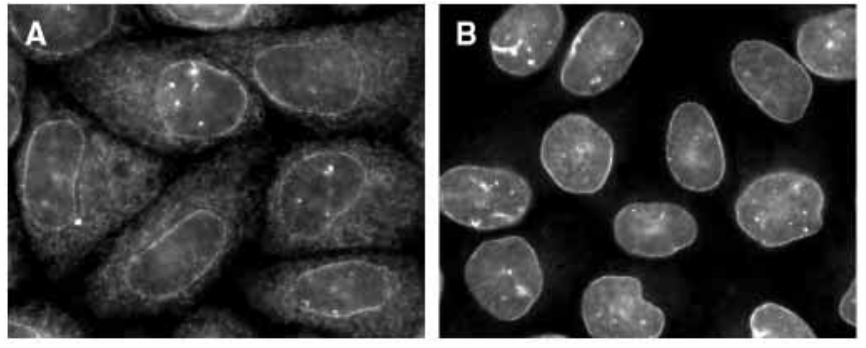

Fig. 2. Silencing of lamin $\mathrm{A} / \mathrm{C}$ in HeLa cells results in displacement of emerin. Cells transfected with lamin A/C siRNA (A) and with luciferase siRNA (B) were stained with emerin antibody. Cells with reduced lamin $\mathrm{A} / \mathrm{C}$ expression (A) show enrichment of emerin in the cytoplasm. Magnification 480×.

with the observation that mice null for $\mathrm{A} / \mathrm{C}$ show normal development and only die one to two months after birth due to muscular dystrophy (Sullivan et al., 1999). Also, lamin A/C is acquired only late in development (Röber et al., 1989). Despite the absence of a growth defect, lamin A/C knockdown cells show a distinct phenotype. Similar to fibroblasts derived from embryos of lamin A/C null mice (Sullivan et al., 1999), lamin $\mathrm{A} / \mathrm{C}$ silenced HeLa cells show an unusual distribution of the inner nuclear membrane protein emerin (Fig. 2). The cytoplasmic distribution of emerin probably within the endoplasmic reticulum is strongly enhanced and supports the view that lamin $\mathrm{A} / \mathrm{C}$ may serve to immobilize emerin. Therefore, knockdown of genes that are not required for cell growth may still cause distinct phenotypes in cultured cells, which can be monitored by appropriate means.
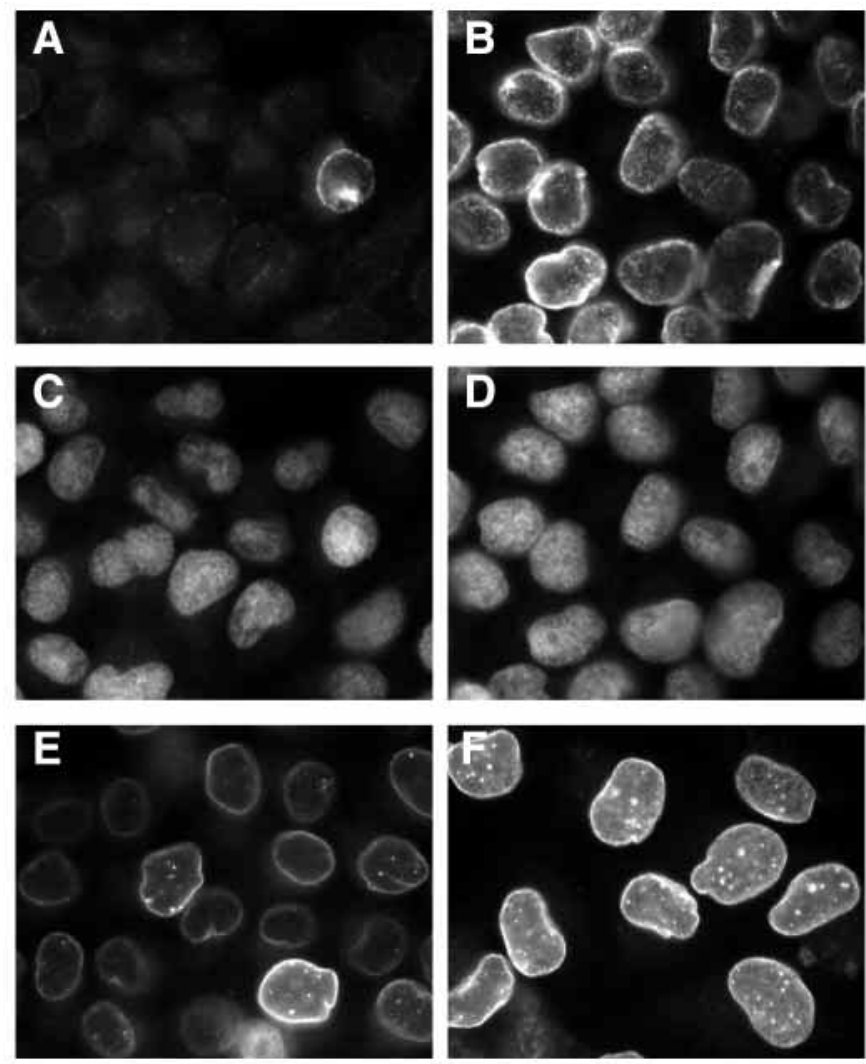
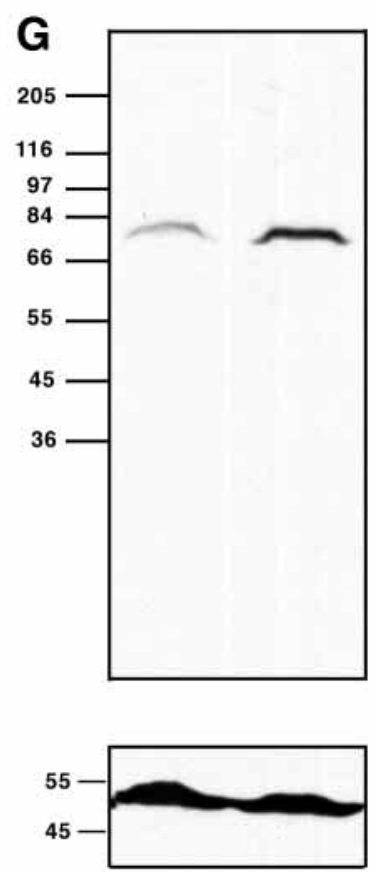

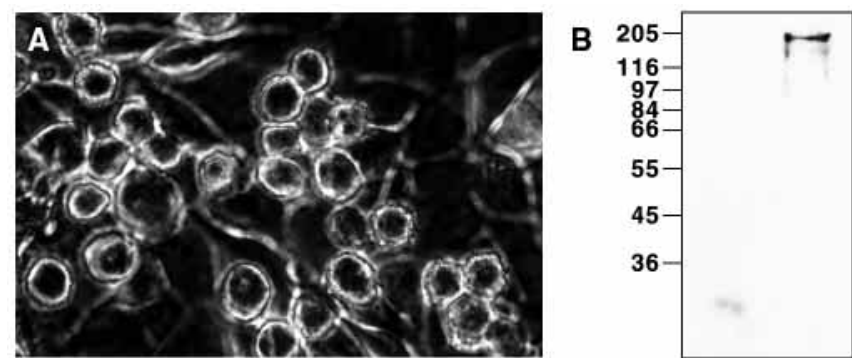

Fig. 4. Silencing of the nuclear pore complex protein Nup153. (A) Phase-contrast of HeLa cells 3 days after transfection with Nup153 siRNA. (B) Western blot of cells transfected with Nup153 siRNA (left) or with luciferase siRNA (right) probed with Nup153specific antibody. Magnification 300× (A).

In contrast to lamin $\mathrm{A} / \mathrm{C}$, whose expression is developmentally regulated, lamin $\mathrm{B} 1$ and lamin $\mathrm{B} 2$ are constitutively expressed (Röber et al., 1989). Lamin B1 and lamin B2 were efficiently silenced 40 hours post-transfection as shown by immunofluorescence or immunoblot (Fig. 3). Later, cells stop growing and become apoptotic. We demonstrated by siRNA-mediated silencing that both B lamins are essential proteins (Table 1). The observed cessation of cell growth upon siRNA transfection indicates that both B-type lamins are necessary to establish a normal nuclear lamina in HeLa cells. Apoptosis induced by lamin B1 and B2 silencing is consistent with the previous finding that perturbation of post-mitotic lamin B1 assembly leads to apoptosis (Burke, 2001). Interestingly transfection with a lamin B1 cDNA carrying a large deletion in the central rod domain leads to lobulated nuclei and growth arrest (Schirmer et al., 2001). These observations do not rule out additional functions for B-type lamins in DNA replication and transcription (Wilson et al., 2001).

The siRNA duplex directed against the nuclear envelope protein LAP2 had no effect on cell growth, yet an effect may have been expected because of the known interaction with the essential B-type lamins (Burke, 2001; Hutchison et al., 2001). Although the LAP2 protein levels were significantly reduced (Table 1), it is unclear whether the residual protein may be responsible for the absence of an effect. Similarly, silencing of the inner nuclear membrane protein emerin, had no effect in HeLa cells. Although lamin A/C knockdown affected emerin localization (see above) (Sullivan et al., 1999), the reverse effect was not observed, and silencing of emerin did not influence the nuclear lamin $\mathrm{A} / \mathrm{C}$ staining pattern.

Nup153 is a protein of the nuclear pore complex and belongs to the F/GXFG family of nuclear pore proteins (Radu et al., 1995). HeLa cells transfected with siRNAs directed against Nup153 rounded up and showed growth arrest (Fig. 4), indicating that this nuclear pore protein is essential.

\section{Knockdown of cytoskeletal proteins}

We previously reported a problem with silencing of vimentin in HeLa cells (Elbashir et al., 2001a) and speculated that this was either due to the abundance of the protein or perhaps due to the sequence selected for siRNA synthesis. We first addressed the issue of abundance and tested siRNAs directed against other major proteins expressed in HeLa cells. Silencing of $\beta$-cytoplasmic actin was readily achieved as monitored by a specific antibody (Fig. 5). Reduction of $\beta$-actin levels led to a
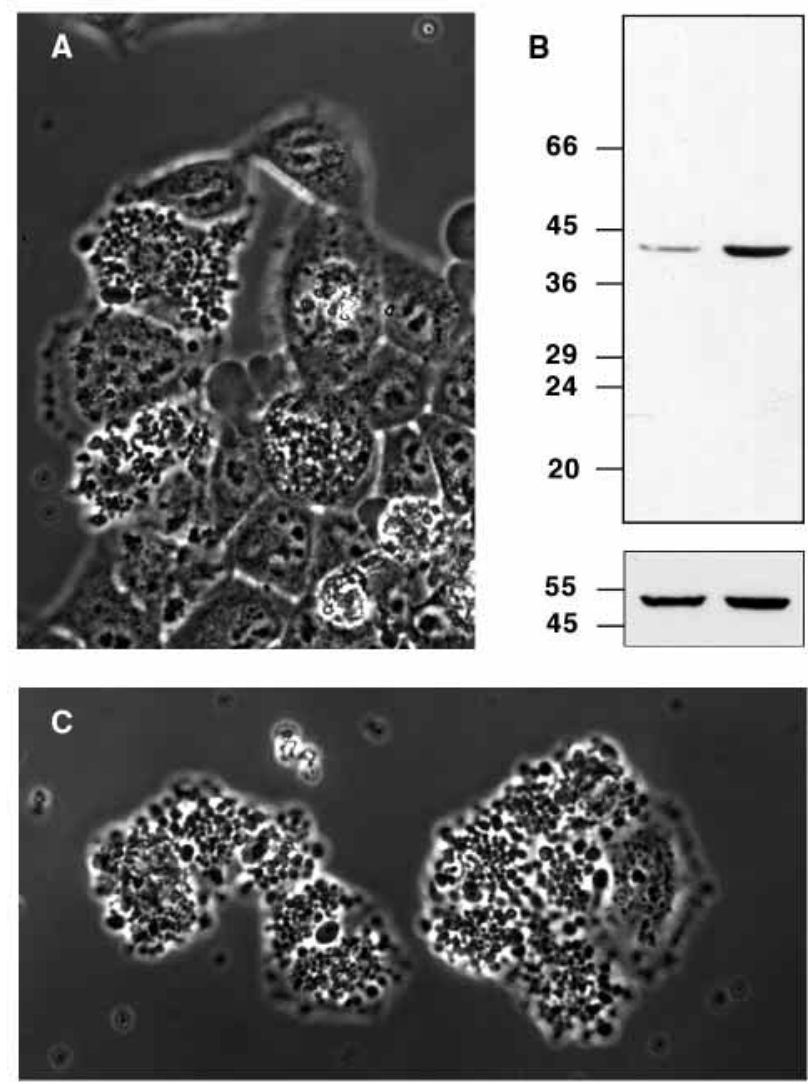

Fig. 5. Silencing of cytoplasmic actins in HeLa cells. (A) Phasecontrast of cells transfected with $\beta$-actin siRNA duplex. Transfected cells show blebbing. (B) Western blot of cells transfected with $\beta$ actin (left) or luciferase (right) siRNA duplexes using $\beta$-actin antibody. The blot was stripped and re-probed with vimentin antibody to check for equal loading. (C) Phase-contrast of cells transfected with $\gamma$-actin siRNA duplex. Transfected cells show a blebbing phenotype similar to cells with $\beta$-actin knockdown. Magnification 480× (A,C).

stop in cell growth and phase microscopy revealed very strong cellular blebbing. The same phenotype was observed after targeting of $\gamma$-cytoplasmic actin, although the precise $\gamma$-actinlevels could not be measured due to a lack of $\gamma$-specific antibody. These results demonstrate that the expression of major cellular proteins can indeed be suppressed by RNAi. Therefore, we tested again for silencing of vimentin with three newly synthesized duplexes directed against different regions of the vimentin mRNA. All newly selected siRNA duplexes were effective in silencing vimentin expression as monitored by immunoblotting. Seventy hours post-transfection, few filaments, which were often fragmented, were detectable by immunofluorescence microscopy (Fig. 6). Some cells contained only short and thin structures, similar to the vimentin squiggles frequently visible in live cells expressing GFPtagged vimentin (Yoon et al., 1998). The absence of a characteristic phenotype after silencing was expected because vimentin null mice develop and reproduce normally (ColucciGuyon et al., 1994). Knockdown cells for another intermediate filament protein, keratin 18, behaved similar to vimentin knockdown cells, and keratin 18 knockout mice also appear normal (Hesse et al., 2000). 

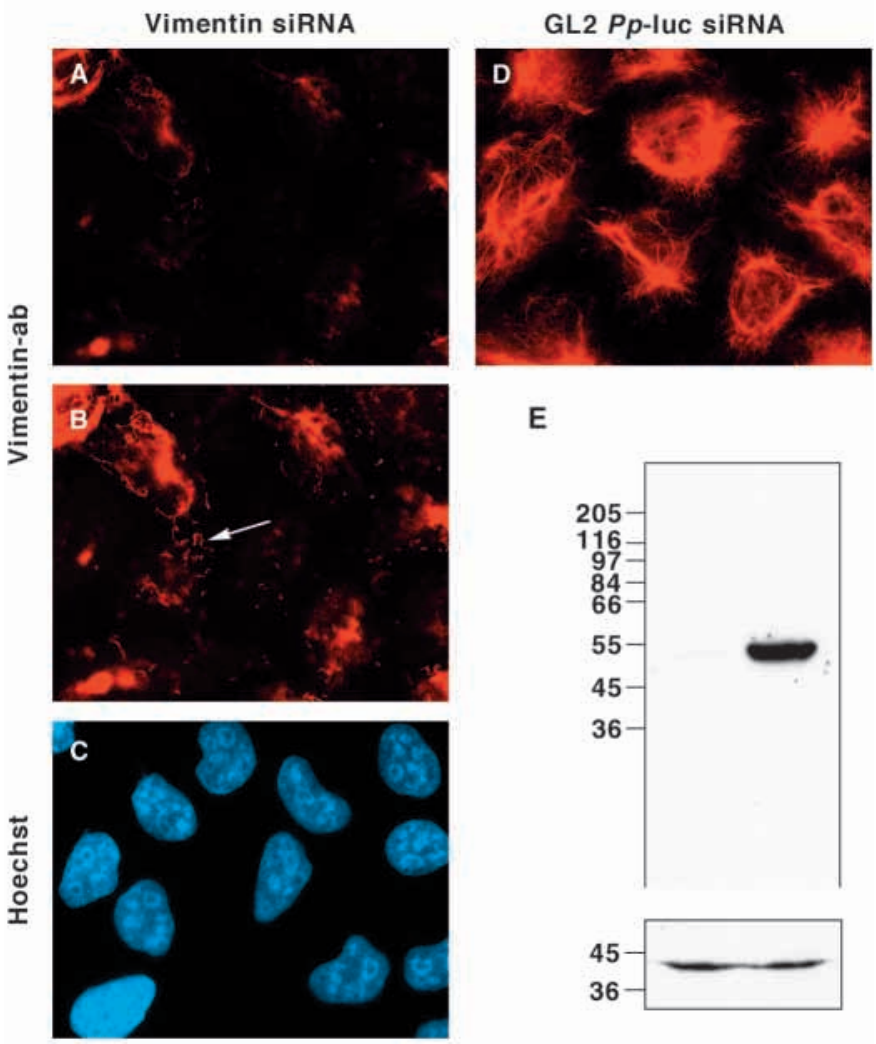

E
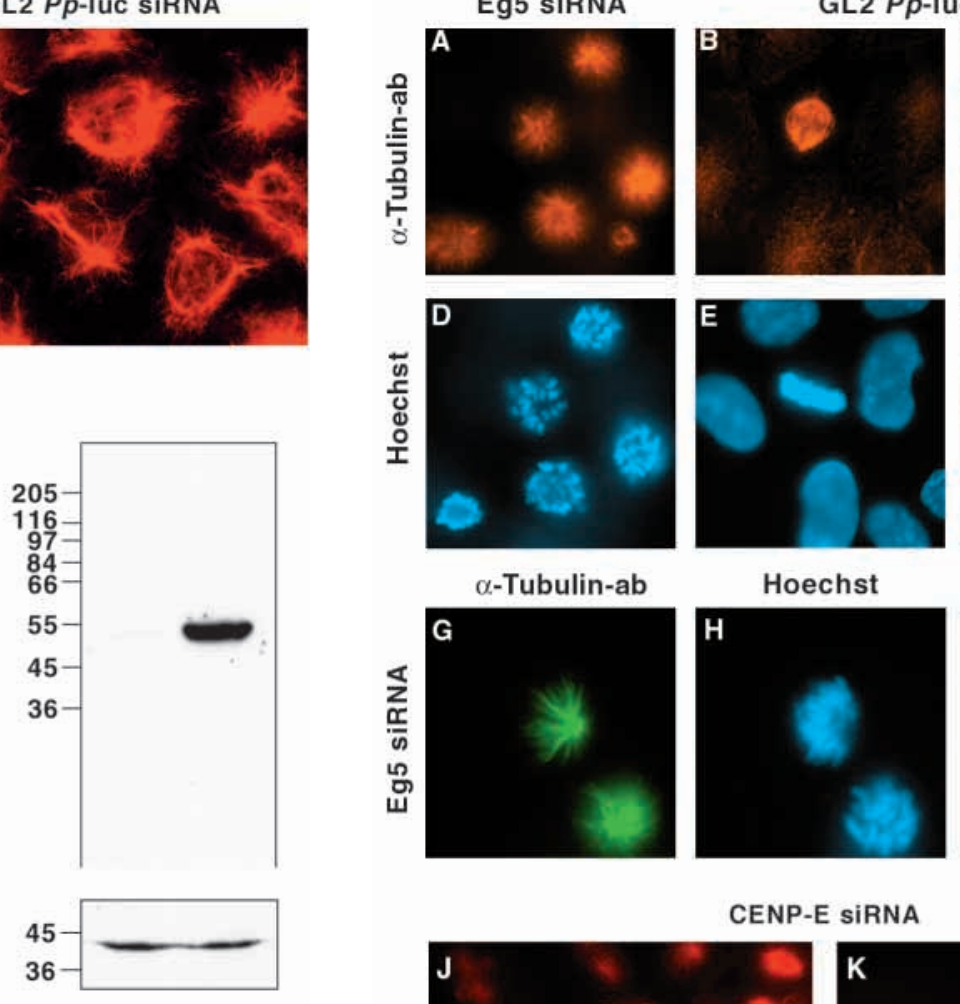

Fig. 6. Silencing of the cytoskeletal intermediate filament protein vimentin. Cells were transfected with vimentin duplex 2 (bp 11451167) (A-C) or with GL2 luciferase duplex (D). (A,B,D) Staining with vimentin V9 antibody; (C) Hoechst staining. Note the strong decrease of vimentin staining in cells transfected with vimentin duplex 2 (A) when compared with the control (D). Only at longer exposure times (B) can short filamentous structures (vimentin squiggles) (arrow) be seen. (E) Western blot using vimentin antibody (top panel), and $\beta$-actin antibody as control (bottom panel). Magnification 460× (A-D).

We then targeted three actin-associated proteins, vinculin, zyxin and VASP, which are usually found in focal contacts (Drees et al., 1999; Critchley, 2000). Successful silencing was documented by immunofluorescence and immunoblotting. Vinculin is essential in mouse 3T3 fibroblasts. Cells became strongly contracted and some cells rounded up and were lost from the substratum. Silencing of zyxin in 3T3 cells is far less dramatic. The cells stayed flat but actin staining with rhodamine phalloidin showed that stress fibers were greatly reduced and focal contacts were diminished. Our vinculin results fit the observation of embryonic lethality in mouse knockout experiments (Xu et al., 1998). VASP is not an essential protein in HeLa cells and mice null for VASP are normal except for a platelet defect (Aszódi et al., 1999). ARC21 is a component of the ARP2/3 complex, which regulates actin filament network formation (Higgs and Pollard, 2001). Using siRNA-mediated silencing we found that ARC21 is an essential protein in HeLa cells (Table 1).

\section{Knockdown of motor proteins}

RNAi was also used to knockdown three microtubuledependent motor proteins (Table 1). The kinesin related motor protein Eg5 was shown earlier to be involved in centrosome
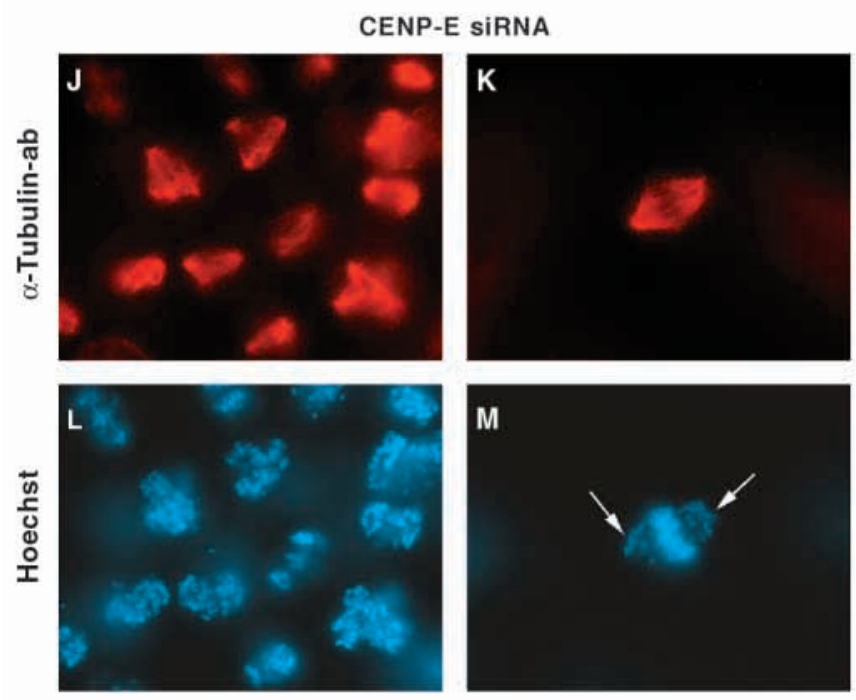

Fig. 7. Silencing of the kinesin-related motor protein Eg5 and of the microtubular motor CENP-E. HeLa cells were transfected with Eg5 siRNA (A,D,G-I) or with luciferase control siRNA (B,C,E,F). Cells are stained for $\alpha$-tubulin (A-C) with a corresponding Hoechst stain (D-F). Cells transfected with Eg5 siRNA are arrested in mitosis and show monoastral microtubular arrays (A,D). By contrast, control cells show normal bipolar spindles in mitosis $(\mathrm{B}, \mathrm{E})$ and microtubule networks in interphase (C,F). A higher magnification of cells transfected with the Eg5 siRNA clearly shows the monopolar spindle $(\mathrm{G})$, which does not overlap with the chromatin staining $(\mathrm{H})$; for overlay see I. HeLa cells were transfected with CENP-E siRNA (J-M). Cells were stained with $\alpha$-tubulin antibody $(\mathrm{J}, \mathrm{K})$ and Hoechst $(\mathrm{L}, \mathrm{M})$. Note the production of multipolar arrays. The higher magnification of a metaphase cell $(\mathrm{K}, \mathrm{M})$ indicates that the loss of CENP-E affects chromosome congression to the spindle equator (arrows). Magnification 480× (A-F,J-M); 750× (G-I).

separation by antibody microinjection experiments (Blangy et al., 1995) and by the use of the specific inhibitor monastrol (Mayer et al., 1999). Targeting of Eg5 by siRNA duplexes 

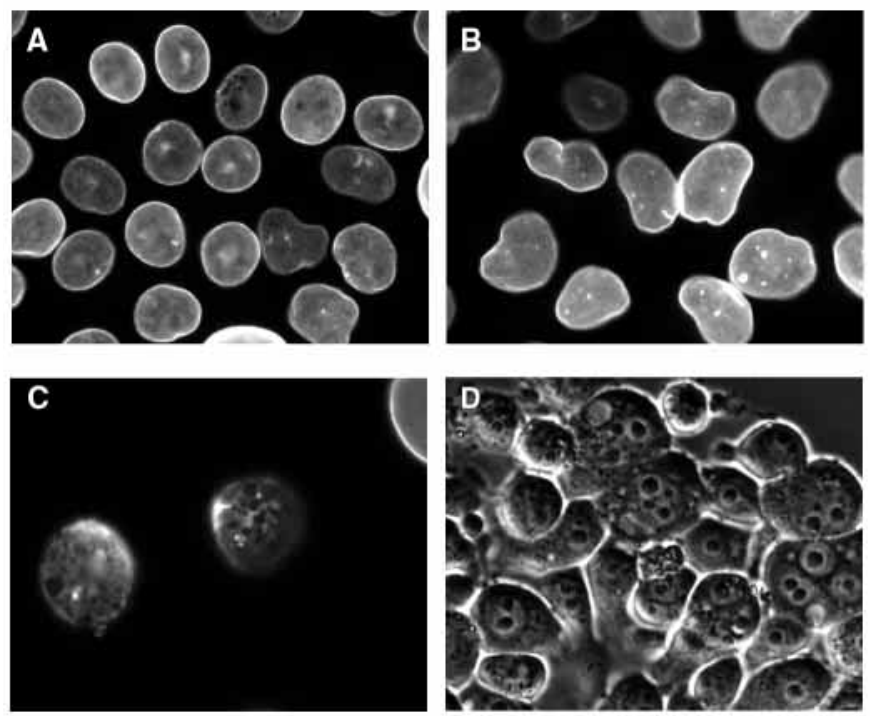

Fig. 8. Silencing of the cyclin-dependent kinase 1 (cdk1). HeLa cells were transfected with cdk1 siRNA (A,C,D) or with luciferase siRNA (B) and stained for lamin A/C (A-C). Cells transfected with cdk1 siRNA round up. Higher magnification shows that the lamina of these cells starts to depolymerize (C). (D) Phase-contrast of HeLa cells 2 days after transfection with cdk1 siRNA. Magnification 480× (A,B); 760× (C); 300× (D).

revealed the formation of half spindles in about $40 \%$ of the arrested cells (Fig. 7) as seen in the previous studies. Because an identical phenotype was observed with respect to previous studies, we did not document Eg5 silencing by immunoblotting. Likewise, the kinetochore-associated motor protein CENP-E was shown to be an essential component of the mitotic spindle, in agreement with recent studies using either antisense-mediated reduction in HeLa cells (Yao et al., 2000) or immunodepletion in Xenopus oocyte extracts (Abrieu et al., 2000). Because of the characteristic aberrant mitotic figures (Fig. 7) we did not attempt to obtain an antibody for immunoblots. Finally, RNAi of cytoplasmic dynein, a protein thought to act in microtubule-kinetochore interactions (Nigg, 2001), also resulted in aberrant mitotic arrest (Table 1).

\section{Knockdown of a mitotic kinase}

The last protein included in our analysis of siRNA-induced phenotypes was the protein kinase cdk1, which is thought to control the M phase of the cell cycle (Nigg, 2001). In contrast to the mitotic motor proteins (see above), the knockdown cells were arrested prior to spindle formation. This is also visible by staining for lamin $\mathrm{A} / \mathrm{C}$, which revealed a thin nuclear rim pattern (Fig. 8). Since phosphorylation of lamins by the cyclindependent kinase cdk1 is thought to lead to the complete disassembly of the lamina (Nigg, 2001) we assume that the partial loss of the lamina observed arises from the reduced amount of cdk1 still present after RNA interference.

\section{DISCUSSION}

The results of our gene targeting experiments by RNAi with 21-nt RNAs in mammalian tissue culture cells document the general applicability of this simple, fast and relatively inexpensive assay of mammalian gene function. Of the 21 genes studied, 13 were shown to be essential for cell growth. For those genes that were previously analyzed for their specific function by other gene targeting methods we find that siRNAinduced gene silencing produced equivalent phenotypes (Table 1).

Our RNAi results on HeLa cells extend the previous characterization of lamin $\mathrm{A} / \mathrm{C}$ to the other two lamins, B1 and B2. Although lamin $\mathrm{A} / \mathrm{C}$ is not an essential protein in the mouse embryo (Sullivan et al., 1999) and in RNAi silenced HeLa cells, the two human B type lamins are now established by RNAi as essential proteins. Interestingly, in the nematode C. elegans there is only a single lamin gene. It encodes a B type lamin that is essential for early embryonic development (Fraser et al., 2000; Liu et al., 2000). Emerin, an inner nuclear membrane protein, is thought to interact with lamin $\mathrm{A} / \mathrm{C}$ (Sullivan et al., 1999) (Fig. 2). We now observe by RNAi that emerin like lamin $\mathrm{A} / \mathrm{C}$ is a nonessential gene in cultured cells. After birth both proteins become essential at least in certain tissues. Mice lacking lamin A/C die after 4-8 weeks due to muscular dystrophy (Sullivan et al., 1999) and three hereditary human diseases (Emery-Dreifuss muscular dystrophy, dilated cardiomyopathy and familial partial lipodystrophy) are connected with missense mutations in lamin A/C (Hutchison et al., 2001; Wilson et al., 2001). Emerin, by contrast, is implicated in X-linked Emery-Dreifuss muscular dystrophy (Blone et al., 1994).

During this study, we experienced in two cases a problem that affected the efficiency of gene silencing. For vimentin and $\mathrm{T}$ antigen we found that the first RNA duplex tested was ineffective, yet already the second duplex directed against a different region of the target resulted in gene silencing. Inspection of the sequences of the ineffective siRNA duplexes did not reveal any unusual feature. These two cases illustrate the value of an antibody to monitor silencing, particularly when no phenotype is observed. Currently we do not know whether the occasional ineffectiveness of an RNAi duplex arises from a local secondary structure of the mRNA, protection of the mRNA by a binding protein, or an as yet unidentified feature in the sequence of the duplex. Alternatively, a minor error in the cDNA sequence or a polymorphism has to be considered, as already a single base change will render the duplex ineffective (S.M.E., unpublished). Since major cellular proteins such as actin, vimentin and keratins can be efficiently silenced in HeLa cells, difficulties for siRNA-mediated gene silencing are only expected when targeting proteins with an unusually long halflife. If the targeted proteins have enzymatic activity rather than structural functions, phenotypes may be more difficult to identify, since siRNA-based technology only provides a knock-down of the targeted protein and not a knockout. However, we note that at least in the case of the cyclindependent protein kinase, cdk1, we readily obtained a premitotic cellular arrest. We also foresee some difficulties when special cell types are used that are difficult to transfect. In HeLa and mouse 3T3 cells as well as rat fibroblasts, we reached transfection efficiencies near $90 \%$, but certain other cell lines may perform poorly in transfection assays. Here other delivery methods for the RNA duplexes such as electroporation and microinjection can be explored. Given the high intracellular stability of siRNA duplexes, we expect that 
RNAi can probably be used in organotypic cultures such as polarized epithelia and muscle cultures, which need a few days to establish. Further improvements of the transfection efficiencies in the future may provide silenced cultures that are suitable as starting material for biochemical analysis.

Our main criteria to identify novel phenotypes after gene silencing were cell growth and light or immunofluorescence microscopy, but in some cases, video microscopy and electron microscopy can be expected to contribute valuable information. Sometimes, additional experiments may document a phenotype even when a nonessential gene is targeted. Thus, silencing of zyxin results in a loss of actin stress fibers and cells lacking lamin $\mathrm{A} / \mathrm{C}$ show misplacement of emerin.

The approach described here is suitable for genome wide analysis of gene function as well as target validation of potentially therapeutic valuable genes. In combination with modern screening methods, one can readily envision high throughput procedures based on siRNAs as a systematic approach to functional genomics of cultured mammalian cells. Based on our results, we anticipate that siRNAs will become a widely used tool for cell biologists to study mammalian gene function, and siRNAs will be particularly useful for the analysis of general cell biological mechanisms such as mitosis, processing and traffic of RNA transcripts, the formation of cellular junctions and membrane trafficking.

We thank H. J. Dehne and W. Lendeckel for expert technical assistance, L. Fredel for help with image processing and B. Burke, W. Deppert, M. Osborn and J. Wehland for antibodies. This work was funded in parts by a BioFuture grant of the BMBF Germany (to T.T.) and the German-Israel Foundation (to K.W.).

\section{REFERENCES}

Abrieu, A., Kahana, J. A., Wood, K. W. and Cleveland, D. W. (2000). CENP-E as an essential component of the mitotic checkpoint in vitro. Cell, 102, 817-826.

Aszódi, A., Pfeifer, A., Ahmad, M., Glauner, M., Zhou, X. H., Ny, L., Andersson, K. E., Kehrel, B., Offermanns, S. and Fässler, R. (1999). The vasodilator-stimulated phosphoprotein (VASP) is involved in cGMP- and cAMP-mediated inhibition of agonist-induced platelet aggregation, but is dispensable for smooth muscle function. EMBO J. 18, 37-48.

Barstead, R. (2001). Genome-wide RNAi. Curr. Opin. Chem. Biol. 5, 63-66.

Bass, B. L. (2000). Double-stranded RNA as a template for gene silencing. Cell, 101, 235-238.

Bass, B. L. (2001). RNA interference, the short answer. Nature 411, 428-429.

Bernstein, E., Caudy, A. A., Hammond, S. M. and Hannon, G. J. (2001). Role for a bidentate ribonuclease in the initiation step of RNA interference. Nature 409, 363-366.

Blangy, A., Lane, H. A., d'Hérin, P., Harper, M., Kress, M. and Nigg, E. A. (1995). Phosphorylation by $\mathrm{p} 34 \mathrm{cdc} 2$ regulates spindle association of human Eg5, a kinesin-related motor essential for bipolar spindle formation in vivo. Cell 83, 1159-1169.

Blone, S., Maestrini, E., Rivella, S., Mancini, M., Regis, S., Romeo, G. and Toniolo, D. (1994). Identification of a novel X-linked gene responsible for Emery-Dreifuss muscular dystrophy. Nat. Genet. 8, 323-327.

Burke, B. (2001). Lamins and apoptosis: a two-way street? J. Cell Biol. 153, F5-F7.

Caplen, N. J., Fleenor, J., Fire, A. and Morgan, R. A. (2000). dsRNAmediated gene silencing in cultured Drosophila cells: a tissue culture model for the analysis of RNA interference. Gene 252, 95-105.

Cogoni, C. and Macino, G. (2000). Post-transcriptional gene silencing across kingdoms. Curr. Opin. Genet. Dev. 10, 638-643.

Colucci-Guyon, E., Portier, M. M., Dunia, I., Paulin, D., Pournin, S. and Babinet, C. (1994). Mice lacking vimentin develop and reproduce without an obvious phenotype. Cell, 79, 679-694.
Critchley, D. R. (2000). Focal adhesions - the cytoskeletal connection. Curr. Opin. Cell Biol. 12, 133-139.

Desai, A., Murray, A., Mitchison, T. J. and Walczak, C. E. (1999). The use of Xenopus egg extracts to study mitotic spindle assembly and function in vitro. Methods Cell Biol. 61, 385-412.

Drees, B. E., Andrews, K. M. and Beckerle, M. C. (1999). Molecular dissection of zyxin function reveals its involvement in cell motility. J. Cell Biol. 147, 1549-1560.

Elbashir, S. M., Harborth, J., Lendeckel, W., Yalcin, A., Weber, K. and Tuschl, T. (2001a). Duplexes of 21-nucleotide RNAs mediate RNA interference in mammalian cell culture. Nature 411, 494-498.

Elbashir, S. M., Lendeckel, W. and Tuschl, T. (2001b). RNA interference is mediated by 21-and 22-nucleotide RNAs. Genes Dev. 15, 188-200.

Fire, A. (1999). RNA-triggered gene silencing. Trends Genet. 15, 358-363.

Fraser, A. G., Kamath, R. S., Zipperlen, P., Martinez-Campos, M., Sohrmann, M. and Ahringer, J. (2000). Functional genomic analysis of Celegans chromosome I by systematic RNA interference. Nature 408, 325-330.

Gonczy, P., Echeverri, C., Oegema, K., Coulson, A., Jones, S. J. M., Copley, R. R., Duperon, J., Oegema, J., Brehm, M., Cassin, E. et al. (2000). Functional genomic analysis of cell division in C-elegans using RNAi of genes on chromosome III. Nature 408, 331-336.

Gueth-Hallonet, C., Osborn, M. and Compton, D. A. (1999). NuMA. In Guidebook to the Cytoskeletal and Motor Proteins (ed. T. Kreis, and R. Vale), pp. 265-268. Oxford: Oxford University Press.

Hammond, S. M., Caudy, A. A. and Hannon, G. J. (2001). Posttranscriptional gene silencing by double-stranded RNA. Nat. Rev. Genet. 2, 110-119.

Harborth, J., Weber, K. and Osborn, M. (2000). GAS41, a highly conserved protein in eukaryotic nuclei, binds to NuMA. J. Biol. Chem. 275, 3197931985.

Hesse, M., Franz, T., Tamai, Y., Taketo, M. M. and Magin, T. M. (2000). Targeted deletion of keratins 18 and 19 leads to trophoblast fragility and early embryonic lethality. EMBO J. 19, 5060-5070.

Higgs, H. N. and Pollard, T. D. (2001). Regulation of actin filament network formation through ARP 2/3 complex: activation by a diverse array of proteins. Annu. Rev. Biochem. 70, 649-676.

Hope, I. A. (2001). Broadcast interference - functional genomics. Trends Genet. 17, 297-299.

Hutchison, C. J., Alvarez-Reyes, M. and Vaughan, O. A. (2001). Lamins in disease: why do ubiquitously expressed nuclear envelope proteins give rise to tissue-specific disease phenotypes? J. Cell Sci. 114, 9-19.

Kim, S. K. (2001). Functional genomics: the worm scores a knockout. Curr. Biol. 11, R85-R87.

Liu, J., Ben-Shahar, T. R., Riemer, D., Treinin, M., Spann, P., Weber, K., Fire, A. and Gruenbaum, Y. (2000). Essential roles for Caenorhabditis elegans lamin gene in nuclear organization, cell cycle progression, and spatial organization of nuclear pore complexes. Mol. Biol. Cell, 11, 39373947.

Maeda, I., Kohara, Y., Yamamoto, M. and Sugimoto, A. (2001). Large-scale analysis of gene function in Caenorhabditis elegans by high-throughput RNAi. Curr. Biol. 11, 171-176.

Matzke, M. A., Matzke, A. J. M., Pruss, G. J. and Vance, V. B. (2001). RNA-based silencing strategies in plants. Curr. Opin. Genet. Dev. 11, 221227.

Mayer, T. U., Kapoor, T. M., Haggarty, S. J., King, R. W., Schreiber, S. L. and Mitchison, T. J. (1999). Small molecule inhibitor of mitotic spindle bipolarity identified in a phenotype-based screen. Science 286, 971-974.

Nigg, E. A. (2001). Mitotic kinases as regulators of cell division and its checkpoints. Nat. Rev. Mol. Cell Biol. 2, 21-32.

Plasterk, R. H. and Ketting, R. F. (2000). The silence of the genes. Curr. Opin. Genet. Dev. 10, 562-567.

Porter, A. (1998). Controlling your losses: conditional gene silencing in mammals. Trends Genet. 14, 73-79.

Radu, A., Blobel, G. and Moore, M. S. (1995). Identification of a protein complex that is required for nuclear protein import and mediates docking of import substrate to distinct nucleoporins. Proc. Natl. Acad. Sci. USA, 92, 1769-1773.

Röber, R. A., Weber, K. and Osborn, M. (1989). Differential timing of nuclear lamin $\mathrm{A} / \mathrm{C}$ expression in the various organs of the mouse embryo and the young animal; a developmental study. Development 105, 365-378.

Schirmer, E. C., Guan, T. L. and Gerace, L. (2001). Involvement of the lamin rod domain in heterotypic lamin interactions important for nuclear organization. J. Cell Biol. 153, 479-489. 
Sharp, P. A. (2001). RNA interference - 2001. Genes Dev. 15, 485-490.

Sijen, T. and Kooter, J. M. (2000). Post-transcriptional gene-silencing: RNAs on the attack or on the defense? BioEssays, 22, 520-531.

Sullivan, T., Escalante-Alcalde, D., Bhatt, H., Anver, M., Bhat, N., Nagashima, K., Stewart, C. L. and Burke, B. (1999). Loss of A-type lamin expression compromises nuclear envelope integrity leading to muscular dystrophy. J. Cell Biol. 147, 913-919.

Svoboda, P., Stain, P., Hayashi, H. and Schultz, R. M. (2000). Selective reduction of dormant maternal mRNAs in mouse oocytes by RNA interference. Development 127, 4147-4156.

Tuschl, T. (2001). RNA interference and small interfering RNAs. ChemBioChem 2, 239-245.

Ui-Tei, K., Zenno, S., Miyata, Y. and Saigo, K. (2000). Sensitive assay of RNA interference in Drosophila and Chinese hamster cultured cells using firefly luciferase gene as target. FEBS Lett. 479, 79-82.

Waterhouse, P. M., Wang, M. B. and Lough, T. (2001). Gene silencing as an adaptive defence against viruses. Nature 411, 834-842.
Wianny, F. and Zernicka-Goetz, M. (2000). Specific interference with gene function by double-stranded RNA in early mouse development. Nat. Cell Biol. 2, 70-75.

Wilson, K. L., Zastrow, M. S. and Lee, K. K. (2001). Lamins and disease: insights into nuclear infrastructure. Cell 104, 647-650.

Xu, W. M., Baribault, H. and Adamson, E. D. (1998). Vinculin knockout results in heart and brain defects during embryonic development. Development 125, 327-337.

Yao, X. B., Abrieu, A., Zheng, Y., Sullivan, K. F. and Cleveland, D. W. (2000). CENP-E forms a link between attachment of spindle microtubules to kinetochores and the mitotic checkpoint. Nat. Cell Biol. 2, 484-491.

Yoon, M., Moir, R. D., Prahlad, V. and Goldman, R. D. (1998). Motile properties of vimentin intermediate filament networks in living cells. J. Cell Biol. 143, 147-157.

Zerrahn, J. and Deppert, W. (1993). Analysis of simian virus 40 small t antigen-induced progression of rat F111 cells minimally transformed by large T antigen. J. Virol. 67, 1555-1563. 